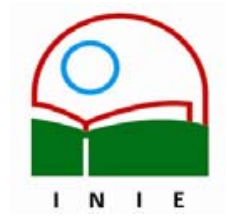

Universidad de Costa Rica

Facultad de Educación

Instituto de Investigación en Educación

ACTUALIDADES INVESTIGATIVAS EN EDUCACION

\title{
LA DIMENSIÓN AMBIENTAL EN EL CURRÍCULO UNIVERSITARIO: UN PROCESO DE CAMBIO EN LA FORMACIÓN PROFESIONAL
}

\author{
ENVIRONMENTAL DIMENSION IN UNIVERSITY CURRICULUM: \\ A PROCESS OF CHANGE IN PROFESSIONAL EDUCATION
}

\author{
Lidia Mayela Hernández Rojas ${ }^{1}$ \\ María de los Ángeles Carrillo Delgado ${ }^{2}$ \\ Claudia Charpentier Esquivel ${ }^{2}$ \\ Olga Emilia Brenes Chacón ${ }^{3}$ \\ Jacqueline García Fallas ${ }^{3}$ \\ Alejandrina Mata Segreda ${ }^{3}$ \\ Claudia Zúñiga Vega ${ }^{4}$ \\ Elizabeth Arnáez Serrano ${ }^{4}$
}

\begin{abstract}
Resumen: La Comisión Interuniversitaria de Educación Ambiental del Consejo Nacional de Rectores de Costa Rica, al completar una década de trabajo, elabora una reflexión sobre su trayectoria y los productos alcanzados. La presente sistematización plantea las diversas áreas de acción en las que su trabajo se ha destacado, aportando información concreta que no solo da fe de su actuación y efectividad, sino que ofrece a quien lee esta propuesta, diversas alternativas para equipos de trabajo que tengan la misión universitaria de transformar la cultura ambiental en sus instituciones y en la formación profesional del estudiantado.
\end{abstract}

Palabras clave: COMISIÓN INTERUNIVERSITARIA DE EDUCACIÓN AMBIENTAL/ EDUCACIÓN SUPERIOR/ EDUCACIÓN AMBIENTAL/

\begin{abstract}
The "Comisión Interuniversitaria de Educación Ambiental" from the Consejo Nacional de Rectores of Costa Rica, by completing a decade of work, reflects about its past work and outcomes. This paper presents the various areas of action in which their work stands out, showing concrete information that not only support its acts and effectiveness but also offers to whoever reads it, different choices for work teams that have the same college mission of transforming the environmental culture in its institutions and in the professional making of students.
\end{abstract}

Key words: COMISIÓN INTERUNIVERSITARIA DE EDUCACIÓN AMBIENTAL HIGHER EDUCATION/ ENVIRONMENTAL EDUCATION/

Las autoras son integrantes de la Comisión Interuniversitaria de Educación Ambiental del Consejo Nacional de Rectores de Costa Rica.

${ }^{1}$ Representante de la Universidad Estatal a Distancia

${ }^{2}$ Representante de la Universidad de Nacional

${ }^{3}$ Representante de la Universidad de Costa Rica

${ }^{4}$ Representante del Instituto Tecnológico de Costa Rica

Artículo recibido: 19 de octubre, 2005

Aprobado: 27 de marzo, 2006 


\section{Introducción}

Para la construcción de la educación superior del futuro en América Latina, se requiere hacer una distinción entre lo que significa la educación superior y lo que conforma la esencia de la universidad. La primera puede concebirse como el ámbito en que se forman cuadros profesionales y técnicos de diversas áreas del conocimiento, al que se accede después de la educación secundaria; la universidad en cambio combina la función de docencia con el desarrollo de la investigación y la creación de la cultura en las disciplinas comprendidas en las ciencias y las humanidades. La universidad se inserta en el conjunto de la educación superior como un patrón de referencia para el resto de las instituciones (Villegas, Sosa, Santamaría y Valdés, 1998). Es su paradigma académico sustentado en tres pilares, a saber, la docencia, la investigación y la acción social, lo que identifica a la institución universitaria. Se le exige un aporte más allá de la mera formación de profesionales, para agregar un elemento cualitativo referido a la incorporación de su propia producción científica en dicha formación, y el fomento de una conciencia social que les lleve a ejercer teniendo como norte el bienestar de la sociedad como un todo.

En el marco anteriormente citado, a la universidad se le plantean grandes desafíos dependiendo del contexto en que ejerce su acción. Jofré (1998) indica que ahora que la humanidad está en capacidad de destruir la Tierra, es indiscutible el papel que la universidad debe asumir en la actualidad y en el futuro para conservar el planeta para las próximas generaciones. Este desafío apenas hace unos años ha sido percibido con claridad por la universidad como institución, obligada de cierta manera por las urgentes reflexiones que en torno a esta situación se han realizado en foros mundiales. Ejemplo de lo anterior es la declaración adoptada por las universidades del mundo en la Conferencia Mundial sobre la Educación Superior de la UNESCO en 1998, en cuya declaración se establece lo siguiente:

La pertinencia de la educación superior debe evaluarse en función de la adecuación entre lo que la sociedad espera de las instituciones y lo que éstas hacen. Para ello, las instituciones y los sistemas, en particular en sus relaciones aún más estrechas con el mundo del trabajo, deben fundar sus orientaciones a largo plazo en objetivos y necesidades sociales, y en particular el respeto de las culturas y la protección del medio ambiente. Fomentar el espíritu de empresa y las correspondientes capacidades e iniciativas ha de convertirse en una de las principales preocupaciones de la educación superior. Ha de prestarse especial atención a las funciones de la educación superior al servicio de la sociedad, y más concretamente a las actividades 
encaminadas a eliminar la pobreza, la intolerancia, la violencia, el analfabetismo, el hambre, el deterioro del medio ambiente y las enfermedades, y a las actividades encaminadas al fomento de la paz, mediante un planteamiento interdisciplinario y transdisciplinario. (UNESCO, 1998, p. 1).

Es así como, de manera previa y posterior a la mencionada declaración, las instituciones universitarias, y en especial las costarricenses, han establecido mecanismos que permiten la concreción del tema ambiental en la formación de sus graduados y graduadas. El presente trabajo es una sistematización de la experiencia concreta construida por las cuatro universidades estatales de Costa Rica, con el propósito de mostrar un esfuerzo factible, pertinente y probablemente replicable en otros países latinoamericanos.

\section{La Comisión Interuniversitaria de Educación Ambiental (CIEA)}

En Costa Rica existen cuatro universidades públicas, a saber: Universidad Nacional (UNA), Universidad de Costa Rica (UCR), Instituto Tecnológico de Costa Rica (ITCR) y la Universidad Estatal a Distancia (UNED), las cuales conforman el Consejo Nacional de Rectores (CONARE), instancia que coordina y articula el quehacer de la educación superior universitaria estatal del país. El artículo 37 del "Convenio de coordinación de la educación superior universitaria estatal en Costa Rica" establece que las instituciones signatarias convienen en establecer una política general de cooperación y de coordinación, y en particular, brindar servicios de apoyo común entre todas estas instituciones. El CONARE ejecuta su quehacer mediante comisiones de trabajo, que integran representantes de cada institución. Existen comisiones integradas por las máximas autoridades universitarias (Rectores y Vicerrectores), las cuales a su vez están divididas en subcomisiones.

Una de las subcomisiones de la Comisión de Vicerrectores de Investigación, que durante 11 años ha trabajado para incluir la Dimensión Ambiental en el quehacer universitario, es la Comisión Interuniversitaria de Educación Ambiental (CIEA), la cual es una muestra de que trabajar con el compromiso de cumplir una meta rompe barreras y causa sinergias entre las personas que han asumido este reto. Esta Comisión tiene como misión "Incrementar la incorporación de la educación ambiental y la Dimensión Ambiental en el quehacer de las universidades públicas costarricenses" (CIEA, 2004), y desde entonces todos sus esfuerzos han sido dirigidos hacia el cumplimiento de esta tarea. Por consiguiente, la CIEA se ha 
comprometido a promover el empoderamiento para la acción, basado en valores y en el desarrollo de una cultura ambiental universitaria.

El concepto orientador de su trabajo, como se expresa en su misión, es el de Dimensión Ambiental, la cual se concibe como un eje transversal articulador del plan de estudios cuya función es enriquecer todo el currículum con un enfoque que propicia valores y conductas en el estudiantado que le permitan abordar constructivamente su relación con el medio que les rodea. Representa una articulación conceptual, metodológica y de significación para la formación profesional, y constituye un punto de encuentro entre diversas ciencias y disciplinas como un fuerte eje de articulación interdisciplinaria que promueve una mayor congruencia de la realidad en la que adquieren nuevos sentidos las ideas y los hechos relacionados con el binomio ser humano - ambiente (Mata, Zúñiga, Brenes, Carrillo, Charpentier, Hernández y Zúñiga, 2003).

Para facilitar la Educación Ambiental de quienes integran las distintas comunidades universitarias, y la inclusión de lo ambiental como una dimensión en el currículum, se ha recurrido a acciones de educación permanente para el profesorado universitario y a la divulgación de publicaciones propias y de otras fuentes. Además se facilitan espacios para la construcción de alternativas metodológicas entre el profesorado universitario de diferentes áreas del conocimiento, y se promueve el establecimiento de alianzas entre el personal interesado en el tema, y con las personas integrantes de la CIEA. Sus tareas impactan la toma de decisiones, la ambientalización del currículo, la elaboración de materiales y el desarrollo de proyectos de investigación.

En varias ocasiones la CIEA ha colaborado con la definición de políticas ambientales y cursos de acción en el plano nacional, trascendiendo hacia ámbitos más amplios de la población. Por ejemplo en la década de los 90 se integró a la Comisión Nacional de Educación Ambiental representando al sector universitario, posteriormente apoyó los esfuerzos para realizar el Plan estratégico de Educación Ambiental del Ministerio de Educación Pública. Actualmente, respondiendo a los acuerdos del Plan Latinoamericano de Educación Ambiental (PLACEA), la CIEA forma parte del grupo costarricense que está elaborando el Plan Nacional de Educación Ambiental (PLANEA), que tiene como propósito recolectar experiencias exitosas y formular un plan maestro de país para los próximos 10 años. Esta es una de las acciones que llevan a cumplir con la misión y visión que enmarcan 
el quehacer de la CIEA; además de difundir y visualizar la incorporación de la Dimensión Ambiental como un eje transversal en otros sectores estratégicos de la sociedad.

$\mathrm{Su}$ trabajo le ha permitido intercambiar experiencias con diferentes organismos e instituciones universitarias del extranjero. La CIEA ha logrado captar el apoyo de ANUIES de México, para la participación de la académica María Teresa Bravo, quien visitó el país para impartir un taller para docentes de las universidades estatales en el que se compartieron y analizaron los acuerdos a los que han llegado todas las universidades mexicanas para incorporar la Dimensión Ambiental. El Dr. Javier García-Gómez de la Universidad de Valencia de España impartió un taller sobre experiencias de esa universidad en el campo ambiental. En estos talleres se ha dado una amplia participación del personal docente de las cuatro universidades estatales costarricenses.

Más recientemente se ha establecido un fuerte vínculo con la Universidad de Illinois, por medio de la profesora M.Sc. Norma Scagnolli. Su campo de especialidad es la educación virtual y su relación con la CIEA se ha concretado en el desarrollo de dos cursos en línea, el primero precisamente sobre este tema, y el segundo sobre materiales educativos para cursos en línea. En este momento las universidades estatales costarricenses junto con la Universidad de Illinois, con la participación también del profesor Dr. Dwane Friend, se encuentran elaborando un proyecto de investigación para la creación de una comunidad de aprendizaje virtual en el campo ambiental, involucrando al profesorado universitario. Otro intercambio de gran valor para la CIEA ha sido el establecido con el Dr. Pedro Rojo de la Universidad de Murcia, España, quien incorporó el tema de la comunicación ambiental al quehacer de la CIEA.

La comunicación ambiental es un recurso necesario para captar la atención de los diversos públicos sobre los problemas ambientales presentes y potenciales y resaltar las oportunidades de un manejo adecuado del ambiente. A nivel universitario es importante lograr la efectividad de las acciones ambientales, por lo que han de identificarse a los actores claves que contribuyan con elementos de juicio y fundamentos racionales en la planificación, puesta en marcha, seguimiento y apoyo a procesos de recuperación, mejoramiento y "gestión racional de los factores que caracterizan el ambiente, el desarrollo a escala humana y la calidad de la vida, con el compromiso y participación activa de todos los miembros de la comunidad" (Tréllez, 1995, p. 133). 
Asimismo la comunicación ambiental, según lo ha identificado Solano (2004) puede contribuir entre otros aspectos a:

1. Desarrollar actitudes en las personas y grupos sociales, basadas en la adquisición de valores sociales que les impulse a participar activamente en la protección y mejoramiento del ambiente

2. Descubrir y cultivar las aptitudes de las personas para resolver problemas ambientales, por sí mismas o actuando colectivamente.

3. Desarrollar la capacidad de evaluación, sentido de responsabilidad, en las personas y grupos sociales, para evaluar los recursos, en función de factores ecológicos, políticos, económicos, sociales, estéticos y educacionales.

Por consiguiente, es importante utilizar el recurso de la comunicación ambiental en la capacitación de la comunidad universitaria que permita contribuir a que la sociedad evalúe su situación ambiental y los factores que la producen y, consecuentemente, adoptar las medidas requeridas para solucionar los problemas ambientales y recuperar el equilibrio dinámico en las relaciones sociedad-naturaleza.

Para la realización de su trabajo, la CIEA ha construido una estrategia particular que integra la planificación y desarrollo de acciones bajo su exclusiva responsabilidad, además de acciones que involucran otras instancias y personas universitarias. Se ha destacado en proyectos de investigación y extensión, entre otras formas, para analizar y proponer alternativas para la inclusión de lo ambiental en el currículum universitario. Además ha concretado mecanismos de articulación con otras instancias universitarias y protagonistas universitarios, y ha establecido planes de acción de corto, mediano y largo plazo. Se detallan a continuación algunas de ellas:

1. Incorporación de miembros del personal académico universitario como aliados y aliadas de la comisión, con el fin de ampliar el grupo de discusión, difundir y multiplicar iniciativas y aumentar el impacto del quehacer de la CIEA.

2. Elaboración de planes estratégicos para la articulación de acciones de la comisión alrededor de metas claras. El primero fue elaborado para el período 2000-2004, y el que se encuentra en vigencia es para el período 2004-2008.

3. Investigación con las autoridades universitarias para definir prioridades en cuanto a los planes de estudio con los que se debería realizar la primera experiencia de incorporación del eje ambiental. 
4. Establecimiento de relaciones con docentes y universidades del extranjero para la realización de investigación e intercambio académico.

5. Búsqueda de financiamiento para proyectos de investigación y formación continua para el profesorado universitario en el campo ambiental.

6. Investigación evaluativa sobre la incorporación de la Dimensión Ambiental en los planes de formación de educadores y educadoras de primaria.

7. Sistematización y divulgación de diversas experiencias de la comisión en foros nacionales e internacionales.

8. Elaboración de los indicadores para la evaluación de la incorporación de la dimensión ambiental en cursos y planes de estudio.

9. Participación de las integrantes de la CIEA en los procesos de incorporación de la Dimensión Ambiental en las universidades estatales.

10. Asesoría de la CIEA en acciones institucionales tendientes a la incorporación de ambiente en políticas y acuerdos universitarios.

11. Empleo de la educación virtual como opción en la formación ambiental del personal docente de las universidades estatales.

12. Formulación de un plan de comunicación para el acercamiento a sectores estratégicos

\section{Participación de CIEA con otros sectores universitarios}

La participación en actividades organizadas por otras comisiones relacionadas con temas afines ha sido fundamental para el acercamiento con los otros grupos de las cuatro universidades públicas costarricenses. Por ejemplo, a finales del 2003 se participó con la ponencia "Valores y Estrategias de Aprendizaje que promueven acciones de los estudiantes a favor del ambiente" en la I Jornada de Experiencias Innovadoras en Educación Universitaria organizada por la Comisión de Vicerrectores de Vida Estudiantil del CONARE (CONARE / Comisión de Vicerrectores de Vida Estudiantil, 2003). Dado su impacto, posteriormente, ésta fue la única ponencia seleccionada para presentarla en una videoconferencia que se realizó en el 2004 con ocasión de la presentación de la memoria de la actividad.

Además dadas las acciones de la CIEA, los Vicerrectores de Extensión y Acción Social integraron a la CIEA en la Expo-Regional Universitaria, donde se divulgó entre las comunidades regionales los resultados del trabajo de investigación realizado sobre el tema de estrategias innovadoras para la incorporación de la Dimensión Ambiental en la formación 
docente (Mata, A.; Zúñiga, C.; Brenes, O.; Carrillo, M.; Charpentier, C.; Hernández, L.; Zúñiga, M.E., 2003)

La Comisión de Vicerrectores de Docencia del CONARE organizó en el 2002 el I Congreso Nacional de Educadores y esta actividad fue considerada por la CIEA como acción estratégica para participar con la ponencia: ¿Conoce los indicadores que hay para medir la incorporación ambiental en los planes de estudio? (CIEA, 2002). Dos años después en un foro similar se presentó el resultado de la investigación sobre incorporación de ejes transversales, que ya ha sido mencionada, y a partir de ahí han surgido invitaciones a la Universidad de Costa Rica y Universidad Estatal a Distancia, pues ya se habían iniciado acciones con las otras dos instituciones que serán mencionadas más adelante.

Asimismo, el taller "Incorporación de la Dimensión Ambiental en los Planes de estudio de las universidades estatales", organizado por la Comisión de Vicerrectores de Docencia del CONARE en octubre de 2004, fue ejecutado por la comisión en vista del currículum acumulado en cuanto a la incorporación de la ambiental como eje curricular. Esta actividad permitió identificar que hay experiencias exitosas en las cuatro universidades estatales sobre incorporación de la Dimensión Ambiental en los planes de estudio y en los cursos de diversas ciencias y disciplinas. Además se facilitó un ejercicio entre quienes participaron, para incluir esa dimensión en sus cursos, concluyéndose que la Dimensión Ambiental se puede implementar en toda la acción docente universitaria de manera transversal.

No obstante que algunas experiencias universitarias demuestran el poco impacto que algunos grupos de trabajo tienen en la transformación de esquemas de trabajo académico, ésta no es la realidad de la CIEA. Cuando en la Universidad Nacional se decidió incorporar varios ejes transversales en los planes de estudio, se dispuso que el equipo fuera coordinado por una de sus representantes de la Comisión. El proceso que se ha seguido se puede consultar en Charpentier y Jiménez (2005). En esta institución se aprobó el sistema de gestión ambiental y el primer compromiso es "Formar profesionales con una clara conciencia y responsabilidad ambiental que se refleje en su desempeño profesional", por esa razón se da una relación directa entre el sistema y el quehacer de la comisión.

Ese mismo reconocimiento a la labor realizada fue la base para participar en un taller del Instituto Tecnológico de Costa Rica sobre la incorporación del eje de ambiente en los planes 
de estudio que se desarrolló en enero del 2004 y donde se expusieron los resultados obtenidos por la CIEA, así como el proceso que se seguía en la Universidad Nacional. Esta participación sirvió de motivación para el trabajo en grupos y en junio de ese año, todos los directores y directoras de unidades académicas presentaron el plan de estudio con este eje incorporado.

Las experiencias de esas dos universidades y la presentación a Vicerrectores de Docencia motivó que se invitara a la Comisión a participar en la reunión de Decanos y Decanas de Facultad de la UCR pues muy concretamente este grupo había solicitado a la autoridad universitaria en el campo de la docencia, que se reforzaran los esfuerzos por incorporar el ambiente como eje transversal en la formación de profesionales de todas las áreas. Esta acción ha sido continuada facilitándose la articulación entre las acciones de la CIEA y las acciones propias de esta universidad. Realizando una tarea similar, se ha participado en el análisis de la incorporación de ejes curriculares en general, en la UNED, donde se brindó apoyo para el trabajo que recién inicia en esa universidad. De esta experiencia la CIEA concluye que una de las instancias universitarias que principalmente facilitan el logro de su misión es la representada por las vicerrectorías de docencia.

Como se indicó en el tema anterior, la CIEA originó la figura de aliados y aliadas de la comisión, personal docente que tiene interés por expandir los hallazgos investigativos y experiencias de esta comisión. Esta relación establecida desde finales de los años 90 ha sido duradera, se ha trabajado de manera empática y coordinando diferentes esfuerzos. Desde el inicio han participado en la mayoría de las oportunidades de actualización que se han desarrollado, algunas personas aliadas han contribuido con las diferentes investigaciones realizadas por la CIEA, y con la difusión de sus propias experiencias en este campo. Se han caracterizado por ser personas influyentes en los procesos desarrollados en las cuatro universidades en el campo ambiental. Este grupo, junto con las integrantes de la comisión, ha desarrollado un sentimiento de pertenencia y solidaridad muy importante para los logros obtenidos.

Por consiguiente los principales logros y conclusiones en el área de participación de la CIEA con otros sectores universitarios, pueden concretarse de la siguiente manera:

1. En todas las universidades estatales existe personal académico con experiencias que de forma creativa y efectiva han logrado incorporar la Dimensión Ambiental en la 
formación profesional. De estas experiencias se ha enriquecido la CIEA para realimentar su trabajo y para hacer evidente que es posible lograr tal incorporación.

2. Como producto de las acciones de articulación con otros sectores académicos, la CIEA ha logrado impactar la transformación curricular y política en cuanto a la incorporación del tema ambiental en las universidades. Existe evidencia que demuestra el uso de un nuevo lenguaje académico y de gestión, que inicialmente ha sido propuesto por esta comisión. Además existen experiencias en diferentes instancias universitarias que de manera explícita han sido originadas por la CIEA.

3. No obstante que los procesos de incorporación del tema ambiental en lo académico, lo político y en la gestión universitaria se han seguido de manera diferenciada en las cuatro universidades estatales en los últimos años, en todos ellos se puede identificar de manera concreta la participación de las integrantes de la CIEA.

4. La CIEA ha sistematizado sus intervenciones al igual que otras instancias universitarias, lo que ha permitido acumular una experiencia útil para continuar con el desarrollo de acciones de incorporación del tema ambiental en el quehacer universitario.

\section{El impacto de la CIEA en el área curricular}

Si bien parte de lo expuesto en el tema anterior está relacionado con el impacto curricular, en este apartado se presentarán experiencias y resultados en los que se explicita este aspecto. La experiencia de la CIEA ha permitido definir un enfoque pedagógico y curricular para la integración de la Dimensión Ambiental en las acciones educativas en la educación superior. Dicho enfoque se explica a continuación mediante el análisis de dos conceptos teóricos que sustentan su trabajo.

La transversalidad se constituye en un enfoque pedagógico y curricular que exige el abordaje de temas y de formas de interacción pedagógica, como unidades integradoras de una visión ética comprometida, en este caso, con la construcción de una mejor relación entre ser humano y ambiente. El reto de esta perspectiva es poder ofrecer una posición humanista y ecológica de la vida en el diseño curricular de los planes de formación de profesionales con el propósito de superar la fragmentación de las áreas del conocimiento. Se busca estimular el desarrollo de capacidades intelectuales a la vez que se internalizan valores y actitudes, en este caso, positivas hacia lo ambiental. La expresión de sentimientos y las maneras de 
entender el mundo en contextos específicos se entrecruzan y se refuerzan mutuamente (Magendzo, Donoso y Rodas, 2000).

La experiencia acumulada mediante la investigación bibliográfica, descriptiva y evaluativa que ha realizado la CIEA en los últimos años, le ha permitido proponer una construcción propia del concepto de Dimensión Ambiental. Mediante esta construcción se busca hacer operativo lo ambiental en términos de transversalidad, concretándose en los ámbitos universitarios desde la docencia, la investigación, la acción social, la administración universitaria y la vida estudiantil. Cada instancia deberá identificar las estrategias concretas que le permitan concretar en su respectiva práctica (académica, administrativa y de vida estudiantil) los principios y aspiraciones que se asuman como guía para la transformación de la cultura ambiental universitaria. Se trata de trascender lo exclusivamente académico para involucrar lo ambiental en el proyecto general de la institución y articularlo con la realidad, con los compromisos que la universidad adquiere con los diversos sectores sociales y con las acciones concretas que realiza (Mata, et al., 2003). Es decir, no solo el currículum universitario se verá modificado por la anterior perspectiva, sino que la dinámica global de las instituciones se transforma incidiendo aún más en la formación de futuros profesionales.

Algunas de las acciones que reflejan lo anterior, y en las que de diversas formas ha estado involucrada la CIEA en las cuatro universidades públicas costarricenses, son las siguientes:

- Diseño de políticas universitarias referidas a lo ambiental.

- Creación de planes de formación de profesionales de diversas disciplinas con la perspectiva ambiental incluida.

- Propuesta de acciones pedagógicas específicas puestas en práctica en los programas de cursos y en las diferentes metodologías desarrolladas por el profesorado de diversas áreas del conocimiento, en busca de implementar lo ambiental en los diferentes cursos universitarios.

- Desarrollo de investigaciones que permitan la inclusión de lo ambiental sea cual sea la ciencia o disciplina desde la que se proponen dichas indagaciones.

- Propuesta de acciones dirigidas a las comunidades en busca de la transformación de conductas ambientalmente inapropiadas.

- Propuesta de mecanismos y procesos de gestión universitaria amigables con el ambiente. 
- Estímulo del desarrollo integral de la población estudiantil buscando la construcción de una mejor relación ser humano - ambiente.

La participación de la CIEA en las anteriores acciones se ha dado de la siguiente manera:

- Asesorías al profesorado universitario e instancias universitarias sobre las perspectivas teóricas y metodológicas que ha desarrollado la CIEA.

- Talleres de reflexión para la construcción de alternativas curriculares para la incorporación de la Dimensión Ambiental.

- Investigación en el campo curricular para la incorporación de la dimensión ambiental.

- Asesoría a trabajos finales de graduación del estudiantado.

A continuación se exponen de manera concreta las participaciones de los últimos 3 años:

\section{Asesorías a profesores universitarios e instancias universitarias.}

Las integrantes de la CIEA prestan su colaboración a diferentes instancias universitarias que se involucran en el tema ambiental. Su participación en estos diversos proyectos puede darse de manera permanente o temporal, dependiendo del propósito que se busque cumplir. En el Cuadro 1 se presentan algunas de las actividades realizadas en los últimos tres años.

\section{CUADRO 1}

Asesorías brindadas por integrantes de la CIEA a profesores e instancias universitarias

\begin{tabular}{|c|c|c|c|}
\hline UNA & UCR & ITCR & UNED \\
\hline $\begin{array}{lr}\text { - Programa } & \text { de } \\
\text { Evaluación } & \text { y } \\
\text { Gestión de la } \\
\text { Calidad Académica } \\
\text { (PEVA), el cual } \\
\text { coincide con la } \\
\text { nueva risión } \\
\text { curricular r que } \\
\text { incorpora cinco ejes } \\
\text { transversales para } \\
\text { la formación de } \\
\text { estudiantes, } \\
\text { incluyendo ambiente } \\
\text { y cultura ambiental. }\end{array}$ & $\begin{array}{l}\text { - Centro de } \\
\text { Evaluación } \\
\text { Académica en cuanto } \\
\text { al eje transversal } \\
\text { ambiente y al tema } \\
\text { general de la } \\
\text { transversalidad. } \\
\text { - Vicerrectoría de } \\
\text { Docencia en cuanto a } \\
\text { la política ambiental } \\
\text { universitaria. } \\
\text { - Participación en } \\
\text { el Programa de } \\
\text { Gestión Ambiental } \\
\text { Integral. }\end{array}$ & $\begin{array}{l}\text { - Centro de } \\
\text { Desarrollo } \\
\text { Académico (CEDA) } \\
\text { - Presentación de } \\
\text { propuesta de de } \\
\text { inclusión del eje } \\
\text { ambiental como eje } \\
\text { transversal en los } \\
\text { currículos del ITCR, } \\
\text { como ponencia que } \\
\text { se presentó y } \\
\text { aprobó en el II } \\
\text { Congreso } \\
\text { Institucional. }\end{array}$ & $\begin{array}{l}\text { Asesoría a la } \\
\text { Carrera de Educa- } \\
\text { ción Preescolar } \\
\text { para la incorpora- } \\
\text { ción del Eje } \\
\text { Ambiental en el } \\
\text { Nuevo plan de } \\
\text { estudios y en las } \\
\text { unidades } \\
\text { didácticas. } \\
\text { Centro } \\
\text { Mejoramiento de la } \\
\text { Producción } \\
\text { Académica para la } \\
\text { incorporación del } \\
\text { eje ambiental en los }\end{array}$ \\
\hline
\end{tabular}




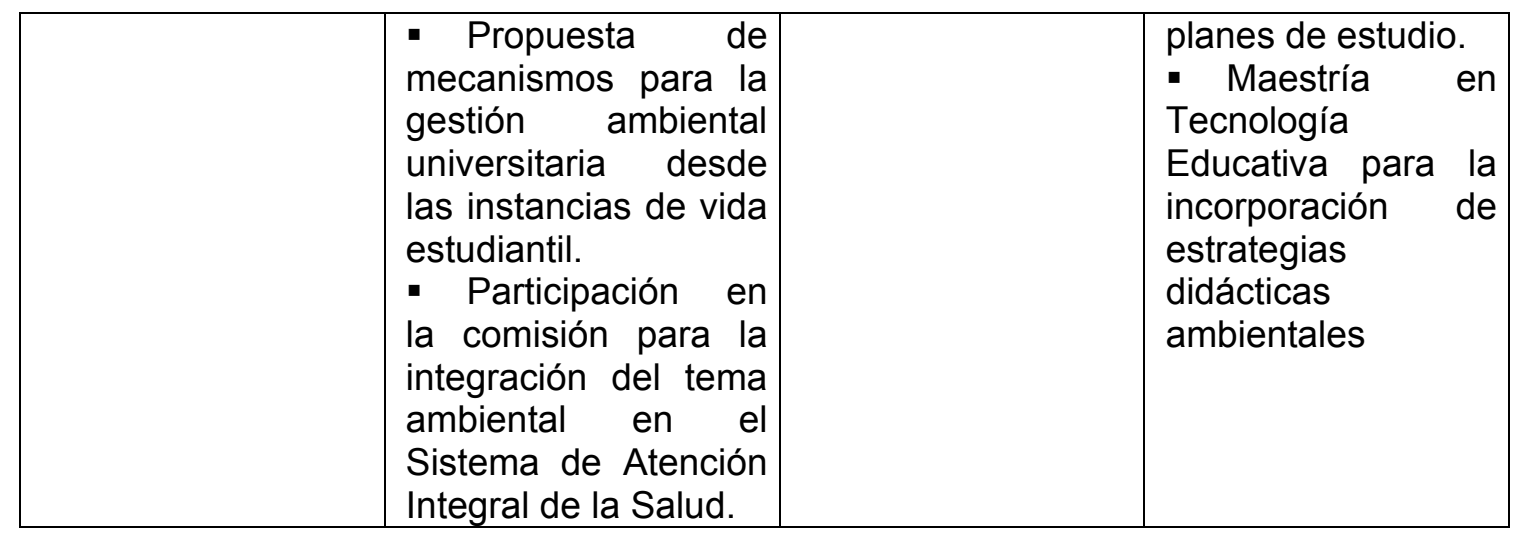

Fuente: elaboración propia 2005.

\section{Talleres de reflexión para la construcción de alternativas curriculares para la incorporación de la dimensión ambiental.}

Otra de las acciones realizadas por la CIEA incluye la organización de talleres que motiven al personal académico a incorporar en su quehacer la Dimensión Ambiental como un eje transversal (Cuadro 2).

\section{CUADRO 2}

Talleres de reflexión para la incorporación de la Dimensión Ambiental organizados por la CIEA

\begin{tabular}{|c|c|c|c|c|}
\hline UNA & & ITCR & & \\
\hline $\begin{array}{l}\text { Foro sobre el } \\
\text { eje ambiente y } \\
\text { cultura } \\
\text { ambiental con } \\
\text { profesores de } \\
\text { las diversas } \\
\text { unidades } \\
\text { académicas de } \\
\text { esta } \\
\text { Universidad. }\end{array}$ & 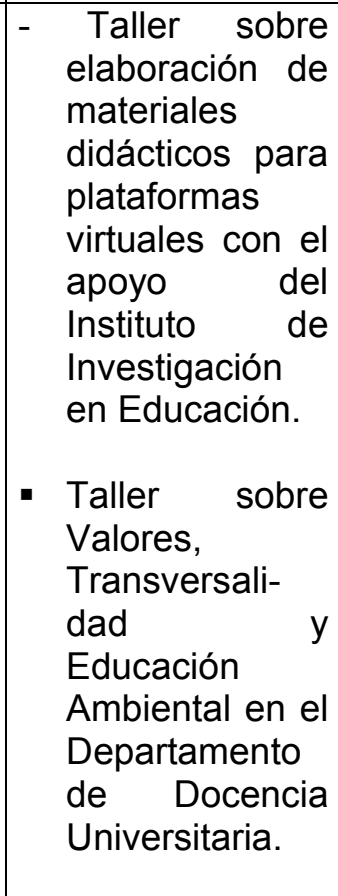 & $\begin{array}{|lr|}\text { - Colaboración } \\
\text { con el taller } \\
\text { para incorporar } \\
\text { la dimensión } \\
\text { ambiental } & \text { en } \\
\text { los planes } & \text { de } \\
\text { estudio } & \text { del } \\
\text { ITCR dirigido a } \\
\text { todos } & \text { los } \\
\text { directores de } \\
\text { las Escuelas. } \\
\text { - Conferencias } \\
\text { en curso de } \\
\text { Comunicación } \\
\text { de } & \text { la } \\
\text { institución } & \\
\text { sobre } & \\
\text { ambiente como } & \\
\text { eje transversal. }\end{array}$ & $\begin{array}{l}\text { - Taller con el } \\
\text { Centro para el } \\
\text { mejoramiento } \\
\text { de la } \\
\text { producción } \\
\text { académica } \\
\text { (CEMPA) para } \\
\text { la capacitación } \\
\text { sobre el eje } \\
\text { ambiental. } \\
\text { - Taller para } \\
\text { compartir } \\
\text { experiencias } \\
\text { sobre la } \\
\text { integración del } \\
\text { eje ambiental } \\
\text { en otras } \\
\text { universidades } \\
\text { en el CEMPA. }\end{array}$ & $\begin{array}{l}\text { Encuentro } \\
\text { interuniversita- } \\
\text { rio en CONARE } \\
\text { con la } \\
\text { Vicerrectorías } \\
\text { de Docencia } \\
\text { para compartir } \\
\text { experiencias } \\
\text { concretas en la } \\
\text { integración de } \\
\text { la EA en los } \\
\text { programas de } \\
\text { cursos. } \\
\text { (participamos } \\
\text { también las } \\
\text { integrantes de } \\
\text { la CIEA con } \\
\text { nuestras } \\
\text { propias } \\
\text { experiencias) y } \\
\text { varias escuelas }\end{array}$ \\
\hline
\end{tabular}




\begin{tabular}{|c|c|c|}
\hline curriculares. & $\begin{array}{l}\text { - Charla sobre } \\
\text { el Día del } \\
\text { Ambiente, en } \\
\text { la Asamblea } \\
\text { Institucional } \\
\text { Representativa }\end{array}$ & $\begin{array}{l}\text { - de la UCR. } \\
\text { - Cursos virtuales } \\
\text { con la } \\
\text { Universidad de } \\
\text { Illinois. } \\
\text { Participación de } \\
\text { la CIEA y otros } \\
\text { profesores de } \\
\text { las } \\
\text { universidades. } \\
\text { - Encuentro } \\
\text { sobre } \\
\text { estrategias } \\
\text { innovadoras } \\
\text { para Trabajo } \\
\text { organizado por } \\
\text { las vicerrectorías } \\
\text { vice Vida } \\
\text { de Estudiantil de } \\
\text { las } \\
\text { universidades } \\
\text { estatales. }\end{array}$ \\
\hline
\end{tabular}

Fuente: elaboración propia 2005.

\section{Investigación en el campo curricular para la incorporación de la dimensión} ambiental.

Como eje generador de conocimiento, la CIEA se ha involucrado en proyectos de investigación propios o participando en otros. A continuación se hará una breve referencia a los mismos.

\section{- Estrategias innovadoras para la formación inicial de educadores en el campo ambiental}

Como proyecto propio cabe destacar el arriba mencionado y que se denomina "Estrategias innovadoras para la formación inicial de educadores en el campo ambiental”, auspiciado por la Coordinación Educativa y Cultural Centroamericana (CECC), instancia centroamericana que integra los ministerios de educación y de cultura de la región (Mata et al., 2003). El propósito de este trabajo fue realizar una investigación evaluativa que permitiera identificar las posibilidades de diferentes estrategias didácticas, como elementos que permiten la incorporación del tema ambiental en diferentes cursos de las carreras de formación docente. Las estrategias probadas son: aula virtual, evaluación por portafolio, generación de 
controversia para la resolución de problemas ambientales, proyecto de investigación, trabajo comunal y mapas conceptuales. Se aplicaron técnicas de evaluación correspondientes a los paradigmas cuantitativo y cualitativo, y se logró identificar las fortalezas de cada una de éstas, concluyéndose que cada una de ellas juega un papel importante en el desarrollo de diferentes destrezas intelectuales, conductuales y afectivas de la población estudiantil que se prepara para la tarea docente. Además del informe de la investigación se elaboró el libro de texto para profesores universitarios denominado "Dimensión Ambiental: Estrategias innovadoras para la formación docente" (Mata, A.; Zúñiga, C.; Brenes, O.; Carrillo, M.; Charpentier, C.; Hernández, L.; Zúñiga, M.E., 2002), editado por la Coordinación Educativa y Cultural Centroamericana para todas las universidades centroamericanas y otros centros de formación de docentes en la región. Constituye el volumen 27 de la Colección Pedagógica Formación Inicial de Docentes Centroamericanos de Educación Primaria o Básica.

Posteriormente, la Universidad Nacional implementó la anterior propuesta de la CIEA, aplicándola en diferentes cursos, e inclusive incorporando otras estrategias bajo la misma perspectiva transversal. En el cuadro 3 se resume la forma en que fue realizada esta actividad:

\section{CUADRO 3}

Incorporación de la Dimensión Ambiental en la UNA, por profesor y unidad temática

\begin{tabular}{|c|c|c|c|c|}
\hline Profesor & $\begin{array}{c}\text { Unidad } \\
\text { Académica }\end{array}$ & Temática & Ejes & $\begin{array}{c}\text { Estrategia de } \\
\text { Aprendizaje aplicada. }\end{array}$ \\
\hline Ileana Vargas & $\begin{array}{l}\text { Educación para el } \\
\text { trabajo }\end{array}$ & $\begin{array}{l}\text { Evaluación } \\
\text { Institucional }\end{array}$ & $\begin{array}{l}\text { Desarrollo } \\
\text { Sostenible }\end{array}$ & Preguntas Intercaladas \\
\hline Humberto Cedeño & \begin{tabular}{l|} 
Medicina \\
Veterinaria
\end{tabular} & Internado Rotatorio & $\begin{array}{l}\text { Ambiente y Cultura } \\
\text { Ambiental }\end{array}$ & $\begin{array}{l}\text { Evaluación } \\
\text { Portafolio }\end{array}$ \\
\hline Guillermo Aguilar & $\begin{array}{l}\text { Escuela } \\
\text { Sociología }\end{array}$ & $\begin{array}{lr}\text { Seguimiento } & \mathrm{y} \\
\text { Evaluación } & \mathrm{de} \\
\text { Proyectos Sociales }\end{array}$ & $\begin{array}{l}\text { Género y Ambiente } \\
\text { y Cultura Ambiental }\end{array}$ & $\begin{array}{l}\text { Proyecto } \\
\text { Investigación }\end{array}$ \\
\hline $\begin{array}{l}\text { Gabriel González } \\
\text { Vega }\end{array}$ & \begin{tabular}{|l|} 
Centro de Est. \\
Generales
\end{tabular} & $\begin{array}{l}\text { Visión Humanista } \\
\text { del Sétimo Arte }\end{array}$ & $\begin{array}{l}\text { Equidad y Ambiente } \\
\text { y Cultura Ambiental }\end{array}$ & Preguntas Intercaladas \\
\hline Iván Sandoval & $\begin{array}{l}\text { Escuela } \\
\text { Ciencias } \\
\text { Biológicas } \\
\end{array}$ & $\begin{array}{lll}\text { Tópicos } & \text { II } & \text { de } \\
\text { biología } & & \\
\end{array}$ & $\begin{array}{l}\text { Desarrollo } \\
\text { Sostenible }\end{array}$ & $\begin{array}{l}\text { Generación } \\
\text { Controversia }\end{array}$ \\
\hline Argenide García & $\begin{array}{lr}\text { Escuela } & \text { de } \\
\text { Topografía } & y \\
\text { Geodesia } & \end{array}$ & Geología & $\begin{array}{l}\text { Ambiente y Cultura } \\
\text { Ambiental }\end{array}$ & Aula Virtual \\
\hline Ana Monge & $\begin{array}{l}\text { Centro de Est. } \\
\text { Generales }\end{array}$ & $\begin{array}{l}\text { Cultura y Adulto } \\
\text { Mayor }\end{array}$ & Género y Equidad & Trabajo Comunal \\
\hline Edgar Núñez & PPS & $\begin{array}{l}\text { Metodología de la } \\
\text { Acción Comunitaria }\end{array}$ & $\begin{array}{l}\text { Género y Ambiente } \\
\text { y Cultura Ambiental }\end{array}$ & Preguntas Intercaladas \\
\hline Diego León López & $\begin{array}{l}\text { Escuela } \\
\text { Psicología }\end{array}$ & Salud y Sociedad & Equidad y género & Organizadores Previos \\
\hline
\end{tabular}




\begin{tabular}{|c|c|c|c|c|}
\hline $\begin{array}{l}\text { Enrique Mata } \\
\text { Selley Ramírez } \\
\text { Mario Oliva }\end{array}$ & $\begin{array}{l}\text { Centro de Est. } \\
\text { Generales }\end{array}$ & $\begin{array}{ll}\text { Ciencia } & \text { y } \\
\text { Tecnología } & \end{array}$ & $\begin{array}{l}\text { Ambiente y Cultura } \\
\text { Ambiental }\end{array}$ & Preguntas Intercaladas \\
\hline Antonieta Corrales & $\begin{array}{l}\text { Esc. De Ciencias } \\
\text { del Deporte }\end{array}$ & \begin{tabular}{|l|} 
Aprendizaje Motor y \\
Fundamentos psico- \\
sociológicos
\end{tabular} & $\begin{array}{l}\text { Género y Ambiente } \\
\text { y Cultura Ambiental }\end{array}$ & \begin{tabular}{|l|} 
Proyecto \\
Investigación
\end{tabular} \\
\hline Julia Rodríguez & $\begin{array}{l}\text { Esc. De Medicina } \\
\text { Veterinaria }\end{array}$ & Embriología & $\begin{array}{l}\text { Desarrollo } \\
\text { Sostenible y y } \\
\text { Ambiente y Cultura } \\
\text { ambiental }\end{array}$ & $\begin{array}{l}\text { Evaluación } \\
\text { Portafolio }\end{array}$ \\
\hline Zaida Fonseca & $\begin{array}{ll}\text { Centro de } & \text { Est. } \\
\text { Generales } & \end{array}$ & $\begin{array}{|ll|}\text { Juventud } & \text { en } \\
\text { América Latina: su } \\
\text { Protagonismo } & \\
\text { Histórico } & \\
\end{array}$ & $\begin{array}{l}\text { Género y Ambiente } \\
\text { y Cultura Ambiental }\end{array}$ & Preguntas Intercaladas \\
\hline Dinia Rojas & $\begin{array}{l}\text { División Educación } \\
\text { Básica }\end{array}$ & $\begin{array}{l}\text { Proyecto educativo } \\
\text { de aula en I y } \\
\text { ciclos }\end{array}$ & $\begin{array}{l}\text { Ambiente y cultura } \\
\text { ambiental }\end{array}$ & \begin{tabular}{|l|} 
Proyecto \\
investigación \\
Evaluación \\
Portafolio, \\
Líneas de vida \\
\end{tabular} \\
\hline
\end{tabular}

Fuente: elaboración propia, 2005.

Asimismo el Instituto Tecnológico de Costa Rica ha tomado la decisión de articular esta perspectiva transversal en diversas actividades académicas y de gestión universitaria, por escuelas. A continuación se presenta un resumen de este esfuerzo.

\section{CUADRO 4}

Incorporación de la Dimensión Ambiental en el ITCR por unidad temática

\begin{tabular}{|l|l|}
\hline \multicolumn{1}{|c|}{ ESCUELA } & \multicolumn{1}{c|}{ ACTIVIDAD } \\
\hline - Escuela de Química & $\begin{array}{l}\text { - ha realizado grandes esfuerzos para } \\
\text { disminuir la cantidad de contaminantes } \\
\text { en sus laboratorios, además utilizan } \\
\text { estrategias para que los estudiantes } \\
\text { cambien sus hábitos en relación con el } \\
\text { ambiente }\end{array}$ \\
\hline - Escuela de Biología & $\begin{array}{l}\text { - imparte la Carrera de Ingeniería en } \\
\text { Biotecnología, tiene el ambiente como } \\
\text { eje transversal en su currículo }\end{array}$ \\
\hline Escuela de Ciencias del lenguaje: & $\begin{array}{l}\text { - ofrece los cursos de Comunicación e } \\
\text { Inglés a todas las carreras, e incluye el } \\
\text { tema ambiental }\end{array}$ \\
\hline - Escuela de Diseño Industrial & $\begin{array}{l}\text { - Elaboración de materiales con } \\
\text { productos de desecho }\end{array}$ \\
\hline Ambiental & $\begin{array}{l}\text { - Además del curso de Fundamentos } \\
\text { de Ecología y Problemática Ambiental, } \\
\text { realiza trabajo comunal enfocado al } \\
\text { ambiente }\end{array}$ \\
\hline
\end{tabular}




\begin{tabular}{|l|lrr|}
\hline - Escuela de Electrónica & $\begin{array}{l}\text { - Desarrollo de proyectos de } \\
\text { investigación con incidencia en la } \\
\text { temática ambiental }\end{array}$ \\
\hline - Escuela de Ingeniería de los Materiales & $\begin{array}{l}\text { - Incorporó un curso sobre } \\
\text { Fundamentos de Ecología y y } \\
\text { Problemática Ambiental }\end{array}$ & \\
\hline
\end{tabular}

Fuente: elaboración propia 2005.

\section{- Indicadores para medir la incorporación de la Dimensión Ambiental en los currículos de las universidades estatales de Costa Rica.}

En el año 2001, mediante un proceso participativo, la CIEA logró construir un sistema de indicadores para identificar qué tan ambientalizados se encuentran los planes de estudios de las diferentes carreras universitarias, tanto a nivel de planificación como de ejecución (CIEA/CONARE, 2002). En cuanto a la planificación se abarcan aspectos relacionados con la presencia de políticas institucionales que incorporan la Dimensión Ambiental, al igual que políticas específicas en las diferentes facultades. Se toman en consideración también aspectos relacionados con la incorporación de lo ambiental en los planes de estudio, explicitados en el fundamento teórico y filosófico de estos planes, en las estrategias didácticas que se propone utilizar y en las formas de evaluación. Además se propone medir la incorporación de lo ambiental en la planificación de acciones para el apoyo logístico a diversas iniciativas amigables con el ambiente. En cuanto a la identificación de iniciativas ambientales en la ejecución de acciones universitarias, el sistema de indicadores propone la medición del grado de cumplimiento en aspectos relacionados con la evolución de las conductas de docentes y estudiantes hacia acciones respetuosas y comprometidas con el ambiente. Para esto se toman en cuenta la inclusión de temas ambientales y estrategias que conlleven prácticas ambientalmente responsables, la participación del personal docente y del estudiantado en proyectos de mejoramiento del entorno en que se encuentran, la observación del estado en que se mantiene el ambiente físico de escuelas y facultades, entre otros. Para cada indicador se establece un nivel de desempeño y se explicitan los medios para la verificación de dicho desempeño.

Este instrumento ha sido utilizado en diversas unidades académicas de las universidades, y la CIEA ha recibido, de quienes lo aplican, recomendaciones para su mejoramiento. Se espera que a mediano plazo el sistema de indicadores sea mejorado y utilizado en las nuevas iniciativas que se ha propuesto desarrollar la comisión a corto plazo. 


\section{- Consulta a académicos universitarios sobre su percepción de la temática ambiental (Barómetro Ambiental)}

El propósito de esta investigación fue determinar áreas prioritarias que requieren ser atendidas mediante proyectos de mejoramiento académico entre el profesorado universitario de las cuatro universidades públicas. El Barómetro Ambiental es el instrumento que permite a la CIEA identificar los temas y estrategias educativas requeridas para ser utilizados en la creación de cursos virtuales y presenciales sobre el tema ambiental, y para el establecimiento de un plan de mejoramiento académico que de manera más estratégica, facilite la incorporación de la Dimensión Ambiental en la formación de profesionales universitarios. Actualmente se cuenta con el primer informe del proyecto (CIEA - CONARE, 2005), el cual contiene los resultado obtenidos de la consulta en las cuatro universidades. Se espera que para el año 2006 ya se tengan los resultados específicos de cada una de ellas.

\section{Trabajos finales de graduación}

Las universidades costarricenses exigen a sus estudiantes la realización de un trabajo final de graduación para completar su formación a nivel de licenciatura y maestría. Las integrantes de la CIEA han asumido como estrategia de trabajo, participar en los comités asesores de estas investigaciones, sobre todo en las dos universidades que forman educadores de manera presencial, la Universidad Nacional y la Universidad de Costa Rica (Cuadro 5).

\section{CUADRO 5}

Trabajos finales de graduación de la UNA y la UCR

donde participaron integrantes de la CIEA

\begin{tabular}{|c|c|}
\hline UNA & UCR \\
\hline $\begin{array}{l}\text { - Ambiente como eje transversal para el } \\
\text { I y ll ciclo de la Enseñanza General } \\
\text { Básica } \\
\text { - Educación ambiental para } \\
\text { guardaparques } \\
\text { - El eje ambiental en los programas de } \\
\text { Estudios Sociales de octavo grado } \\
\text { - Ejes transversales en la educación } \\
\text { matemática } \\
\text { - Incorporación de ambiente en } \\
\text { escuelas unidocentes cercanas al } \\
\text { parque Nacional Palo Verde }\end{array}$ & $\begin{array}{l}\text { - Capacitación de profesores de la carrera } \\
\text { de preescolar para la inclusión del tema } \\
\text { ambiental en sus cursos universitarios } \\
\text { - La orientación y el eje transversal de } \\
\text { valores en la Educación Secundaria } \\
\text { costarricense (incluye el tema } \\
\text { transversal Cultura ambiental y } \\
\text { desarrollo sostenible). } \\
\text { - Estudio de especies en peligro de } \\
\text { extensión para la Educación Preescolar. } \\
\text { - Educación Ambiental y Relaciones } \\
\text { Internacionales. }\end{array}$ \\
\hline
\end{tabular}


- Plan estratégico de educación ambiental para la Empresa de Servicios Públicos de Heredia

- Adopte una quebrada en 5 colegios del país.

- Plan de manejo de desechos sólidos para la Empresa de Servicios Públicos de Heredia.

Fuente: elaboración propia, 2005.

\section{Interacción con otras instancias universitarias para el enriquecimiento en el campo de la docencia.}

Para la elaboración de su primer plan estratégico, la CIEA debía identificar un camino a seguir delimitando su acción a aquellas áreas del conocimiento que parecían ser más propicias y de urgente atención. Así fue como, con el apoyo de todas las autoridades universitarias, pero en especial de las autoridades del área de docencia, se determinó que los esfuerzos planificados debían concentrarse en las carreras de educación por ser sus graduados quienes tenían mayor capacidad de proyección, y para luego facilitar a otras carreras experiencias innovadoras. La CIEA reconoce que el trabajo a partir de las bases (el profesorado universitario), puede verse ricamente ampliado mediante la toma de decisiones de autoridades universitarias. Si bien esta comisión está adscrita a los Vicerrectores de Investigación de las universidades del CONARE, se ha identificado como aliado principal a los Vicerrectores de Docencia por la naturaleza del objeto de su acción. Además ha utilizado la estrategia de intercambiar conocimiento y experiencias entre universidades con la participación de sus integrantes indistintamente de la universidad a la que pertenecen. A lo largo de los últimos tres años la CIEA ha intercambiado ideas y apoyado iniciativas de diversas instancias universitarias como las vicerrectorías en general, y otras instancias de autoridad como los Consejos Universitarios y oficinas administrativas, además de centros de investigación.

En el Cuadro 6 se destacan algunos de los esfuerzos desarrollados en este campo. 


\section{CUADRO 6}

Interacción de las integrantes de la CIEA con diferentes autoridades universitarias.

\begin{tabular}{|c|c|c|c|}
\hline UNA & UCR & ITCR & UNED \\
\hline 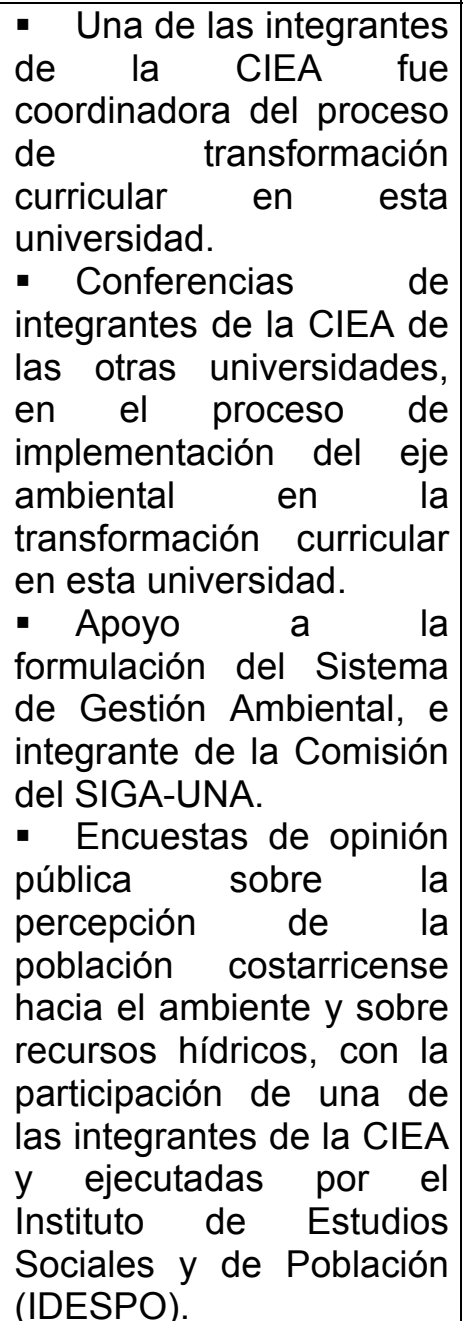 & 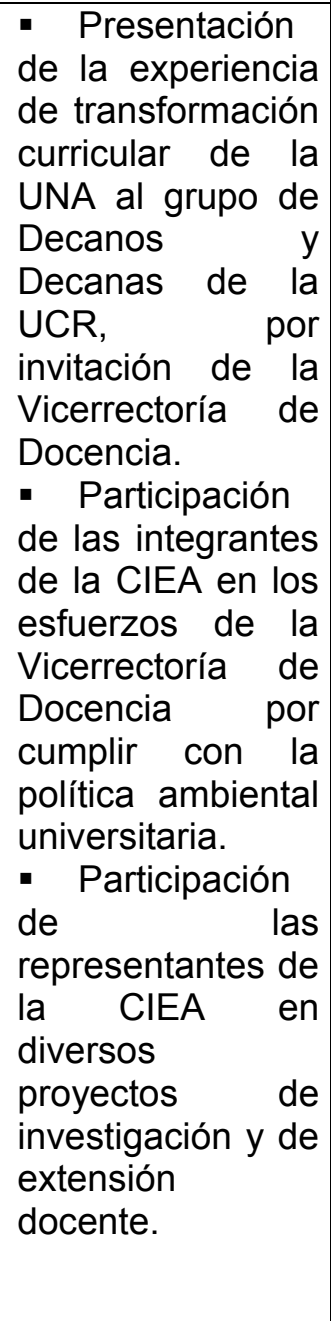 & $\begin{array}{lr} & \text { Participación } \\
\text { de integrantes de } & \\
\text { la CIEA de otras } \\
\text { universidades en } \\
\text { la reunión } & \text { con } \\
\text { directores } & \text { de } \\
\text { Escuelas } & \text { y } \\
\text { director } & \text { del } \\
\text { Centro } & \text { de } \\
\text { Desarrollo } & \\
\text { Académico } & \text { de } \\
\text { este instituto. } & \text { Se } \\
\text { logró que } & \text { los } \\
\text { docentes } & \\
\text { presentaran } & \text { una } \\
\text { propuesta } & \text { de } \\
\text { inclusión de } & \text { la } \\
\text { Dimensión } & \\
\text { Ambiental en sus } \\
\text { cursos. }\end{array}$ & $\begin{array}{l}\text { - Participación } \\
\text { de integrantes de } \\
\text { la CIEA de otras } \\
\text { universidades en } \\
\text { reuniones con } \\
\text { representantes } \\
\text { del área docente } \\
\text { de esta } \\
\text { universidad }\end{array}$ \\
\hline
\end{tabular}

Fuente: elaboración propia, 2005.

\section{Elementos que contribuyen a la evaluación de la pertinencia y efectividad de la CIEA}

La CIEA cuenta con una trayectoria de más de 10 años. Mucha evidencia se ha recogido en el camino, que le permite concluir que su trabajo ha sido provechoso para el enriquecimiento de la formación de la población estudiantil universitaria. Anualmente se realiza un análisis cuidadoso de sus logros de acuerdo con el plan estratégico quinquenal, y los planes operativos anuales. Estos logros se hacen evidentes en los informes de labores que cada año se presentan a la Comisión de Vicerrectores de Investigación del CONARE. Otros 
informes se han elaborado, referidos a actividades especiales realizadas como proyectos académicos de la comisión. El más reciente de ellos se denomina "Actualización de la CIEA en Educación Virtual para incorporar ambiente en los planes de estudio universitario" (CIEA/CONARE, 2005). Constituye el informe final de un proyecto académico auspiciado por la Fundación CR-USA y reporta logros sobre cinco diferentes componentes:

- Detección de las fortalezas y debilidades de las integrantes de la CIEA en el campo de la Educación Virtual.

- Plan de capacitación virtual para el profesorado universitario.

- Sistematización de la experiencia educativa: Curso Virtual "Preparación de materiales para cursos en línea".

- Producción de materiales virtuales producto del taller exploratorio.

- Perfil del proyecto para la National Science Foundation.

No obstante lo anterior, se hace necesario realizar una evaluación más sistemática del quehacer de la CIEA, por lo que se ha considerado la posibilidad de desarrollar un proyecto basado en la propuesta teórica y metodológica de evaluación de Atkinson (en Badilla et.al., 1998) que consiste en la identificación de datos de proceso o transaccionales, referidos a actividades realizadas, y de producto. Estos datos serían recolectados mediante estrategias cuantitativas y cualitativas que permitan a la CIEA responderse a la pregunta de qué tan efectivo ha sido su trabajo. Un primer paso para la realización de esta evaluación ya se ha dado y se encuentra presente en el plan operativo del año 2006, consistente en la recolección del número y calidades de las personas participantes en las distintas actividades desarrolladas por la CIEA en el quinquenio 2004-2008.

\section{Epílogo}

La Comisión de Vicerrectores de CONARE conformó la Comisión Interuniversitaria de Educación Ambiental (CIEA), hace más de diez años, esto ha permitido que las cuatro universidades estatales trabajen unidas para incorporar la Dimensión Ambiental como eje transversal en su quehacer. La conformación de esta comisión ha permitido potenciar las acciones tales como: inclusión del eje ambiental dentro de las políticas de las diferentes instituciones, talleres, cursos, asesorías, investigaciones y otros. Además, las integrantes de esta comisión se caracterizan por apoyarse mutuamente para contribuir con el cumplimiento de la misión en cada una de las cuatro universidades estatales. Esto no hubiera sido posible si cada una hubiera actuado en forma independiente en su ambiente laboral. 
Cabe resaltar que la motivación que caracteriza a esta comisión es uno de los elementos fundamentales que guía su accionar y a la vez que le imprime dinamismo a esta labor. Lo más importante es que las integrantes disponen de un tiempo mínimo asignado para estas labores o del todo no disponen de tiempo, sin embargo, se demuestra que con voluntad y entrega se pueden lograr objetivos tan importantes como los de la CIEA.

De acuerdo con los retos que la sociedad actual ha planteado a las universidades públicas, el presente esfuerzo, aunado a los diferentes proyectos que en otros campos desarrolla cada universidad por separado, podrían hacer una diferencia en el futuro para las nuevas generaciones. Las personas que desarrollan una tarea profesional asumen una tarea protagónica en el desarrollo humano y ambiental sostenibles pues mediante su conocimiento teórico y práctico están en capacidad de proponer y ejecutar acciones responsables sobre la base de la ciencia y la tecnología, fundadas en otra ética ambiental, aquella que vela por la vida, no por la destrucción.

\section{Referencias}

Charpentier, C.; Jiménez, L. (en prensa). La inserción de ejes transversales en la Universidad Nacional es un proceso flexible y participativo. Contexto normalista.

Badilla, R.; Coto, A.; Rodríguez, F.; Turcios, M.G. (1998). Propuesta de evaluación desde un enfoque de planificación para que los orientadores valoren el impacto de sus acciones y su quehacer profesional en las instituciones educativas públicas, diurnas, de Tercer Ciclo y Educación Diversificada. Tesis para optar al grado de Licenciatura en Ciencias de la Educación con énfasis en Orientación, Universidad de Costa Rica.

Comisión Interuniversitaria de Educación Ambiental (CIEA). (2002). Indicadores para medir la incorporación de la dimensión ambiental en los currícula de las Universidades Estatales de Costa Rica. San José: CONARE.

Comisión Interuniversitaria de Educación Ambiental (CIEA). (2004). Plan estratégico 20042008. Material no editado, disponible en OPES/CONARE.

Consejo Nacional de Rectores (CONARE). (1990) "Convenio de coordinación de la educación superior universitaria estatal en Costa Rica". Leyes, convenios y decretos de la Educación Superior Universitaria Estatal en Costa Rica. San José: OPES/CONARE.

Consejo Nacional de Rectores (CONARE) / Comisión de Vicerrectores de Vida Estudiantil (2003) Memoria de la I Jornada de Experiencias Innovadoras en Educación Universitaria. Material disponible en CD. 
Jofré, A. (1998). La universidad en América Latina. Cartago: Editorial Tecnológica de Costa Rica.

Magendzo, A.; Donoso, P.; Rodas, M.T. (2000). Los objetivos transversales de la educación. Santiago de Chile: Editorial Universitaria.

Mata, A.; Zúñiga, C.; Brenes, O.; Carrillo, M.; Charpentier, C.; Hernández, L.; Zúñiga, M.E. (2002). Dimensión Ambiental: Estrategias innovadoras para la formación docente. San José: CECC.

Mata, A.; Zúñiga, C.; Brenes, O.; Carrillo, M.; Charpentier, C.; Hernández, L.; Zúñiga, M.E. (2003). Estrategias innovadoras para la formación inicial de educadores en el campo ambiental. San José: CECC.

Trellez, E. y Quiroz, C. (1995). Formación ambiental participativa. Lima, CALEIDOSOEA.

Solano, David. (2004). Comunicación y generación de conciencia ambiental. Recuperado el 26 de abril de 2005, de http://www.conam.gob.pe/modulos/home/comunicacion.htm

UNESCO. (1998). La educación superior en el siglo XXI. Visión y acción. París: Conferencia Mundial sobre la Educación Superior, UNESCO.

Villegas, A.; Sosa, M.; Santamaría, R.; Valdés, B. (1998). Políticas y estrategias para la universidad latinoamericana del futuro. México: Unión de universidades de América Latina, UDUAL. 